\title{
Exploration on the Construction of Innovative Modern Circulation Virtual Simulation Experimental Teaching Centers: Taking Shanghai Business School as an Example
}

\author{
Xu Wen ${ }^{\mathrm{a}}$, Wu Qianyun \\ National Demonstration Center for Experimental Modern Circulation Education (Shanghai Business School), \\ No.123, Fengpu Avenue, Shanghai, 201400, China \\ asbstracy2009@163.com, buqy@sbs.edu.cn
}

Keywords: Modern Circulation; Innovation; Virtual Simulation; Experimental Teaching

\begin{abstract}
Based on the requirements of the Ministry of Education for constructing national virtual simulation experimental teaching centers and complying with the development of innovative experimental teaching, this paper explores the construction of innovative modern circulation virtual simulation experimental teaching centers from many aspects including the design of virtual simulation experimental teaching system, co-construction by university-enterprise cooperation, sharing of teaching resources, construction of teaching platforms and so on, and studies the reform mode of experimental teaching under the environment of "Internet+".
\end{abstract}

The construction task of virtual simulation experimental teaching centers is to realize the teaching functions which real experiments do not possess or find difficult to complete. Reliable, safe and economic virtual simulation experimental projects can be provided when it comes to high-risk or extreme environments, unreachable or irreversible operation, high-cost, high-consumption, large or combined trainings and so on. ${ }^{1}$ Virtual simulation experimental teaching relies on virtual reality, multimedia, human-machine interaction, database, network communication and other technologies and constructs a highly simulated virtual experimental environment and experimental subjects. Students carry out experiments in the virtual experiment, which achieves the teaching effect required by teaching syllabus.

In the process of being open to the experimental teaching and practical training of all majors related to commercial circulation for many years, Modern Circulation Virtual Simulation Experimental Center of Shanghai Business School adheres to the guiding thought of "highlighting features, sharing resources, emphasizing application, combining virtuality with reality and taking the lead in technology". It takes advantage of professional simulation software and home-made virtual simulation experimental system according to the experimental content which real experimental platforms find difficult to carry out with high complexity and cost, long cycle and a wide range in the process of practical classroom teaching and experiments. The center also constructs a three-dimensional and multi-layered virtual simulation experimental platform with a high sense of reality and accuracy and facing all majors related to the commercial circulation of the whole school based on network cloud computing and informatization platforms.

\section{Necessity of constructing modern circulation virtual simulation experimental teaching centers}

At present, Shanghai is in an important period of accelerating the transformation of economic development mode and adjusting industrial structure. The construction and development of "China (Shanghai) Pilot Free Trade Zone”, “International Finance Center”, “Shoppers’ Paradise” and "Smart City" put forward new requirements on the cultivation of high-quality talents in commercial circulation. To adapt to the new needs and normal of business development in this area, Shanghai

${ }^{1}$ [1] Notice on Carrying out the Construction Work of National Virtual Simulation Experimental Teaching Centers, Jiaogaosihan [2013] No. 94 
Business School abides by the school-running goal of cultivating applied talents in business operation and management and business culture construction and takes solving practical problems in the commercial circulation industry as traction to optimize the structure of disciplines and majors and improve school-running characteristics.

With the extensive application of modern information technologies like Internet and mobile terminals, new business models have constantly sprung up, which creates new commercial forms. The fusion of "four flows" including business flow, logistics, capital flow and information flow becomes the new feature of modern commercial circulation industry. Restricted by equipment and software, traditional experiments in economic management are confined to the simple repetition and verification of business process and lack the training of comprehensive application and innovation capability of students due to single form and dispersed and isolated knowledge structure. It is pressing to develop virtual simulation experimental technologies, break through the space-time limitation of existing experimental resources and make full-scale experiments and practice complement each other.

The construction of modern circulation virtual simulation experimental teaching centers can solve the following three concrete problems.

Firstly, the traditional practical teaching methods of internship in enterprises find it difficult to achieve the practical teaching goal of cultivating the application ability and overall industry cognition of students in key positions.

Secondly, it is difficult for theoretical teaching and full-scale experiments to reproduce economic management activities in business environment due to the complexity of economic management activities in business enterprises. In the meanwhile, the impact of extreme cases and emergencies in business environment makes teaching activities unable to reproduce systematic observation through real experiments.

Thirdly, it takes a lot of money and time to cultivate the innovation and entrepreneurship ability of students. The implementation of real entrepreneurial projects is usually accompanied by numerous efforts, high risk, limited scope of knowledge and insufficient technical capacity. Entrepreneurship may be blind and random to a great extent.

The construction of centers aims to solve the contradiction between the theoretical teaching of the commercial circulation industry and the cultivation of students' application and innovation capability and improve the actual effect of experimental teaching. Based on existing classroom theoretical teaching, professional practice, social practice and full-scale experiments, the construction of centers takes into account real position skills, business environment and data, decision-making process and complete business ecosystem, carries out modeling and simulation for key links, combines with multimedia and Internet technology and constructs virtual simulation experimental teaching platforms possessing a high sense of reality and suiting the characteristics of experimental teaching, which is the beneficial complement and innovation of practical teaching.

\section{Thought of constructing modern circulation virtual simulation experimental teaching centers}

(1) Highlight the industry characteristics of commercial circulation, combine with the goal of ability cultivation and construct multi-layered virtual simulation experimental platforms integrating application and innovation.

(2) Display the advantage of cooperation between applied universities and enterprises, transform enterprise resources into virtual simulation experimental teaching content and construct virtual simulation experimental platforms for practical application.

(3) Rely on the existing experimental teaching basis of cross-school cooperation, highlight the sharing of superior experimental resources and construct cross-university and cross-regional comprehensive virtual simulation experimental platforms.

(4) Combine virtual simulation experimental technologies with experimental content organically and construct the virtual simulation experimental platforms of "combining virtuality with reality, complementing each other and emphasizing reality rather than virtuality”. 
(5) Make the most of the construction foundation of smart campus in schools, combine with the high and new technology of virtual simulation experiments and construct intelligent, open and virtual simulation experimental platforms.

\section{Practical exploration of constructing modern circulation virtual simulation experimental teaching centers}

\subsection{Construction goal of virtual simulation experimental teaching centers}

The construction of Modern Commercial Circulation Virtual Simulation Experimental Center of Shanghai Business School focuses on the industry characteristics of commercial circulation, gives play to discipline advantages, strengthens university-enterprise and university-university cooperation, adheres to the teaching concept of "focusing on the industry, integrating industry, education and research, centering on application and stimulating innovation”, insists on the principle of "combining virtuality with reality, complementing each other and emphasizing reality rather than virtuality" and strives to make breakthroughs and innovations in virtual simulation experimental project library, cross-school sharing of experimental resources, management of intelligent experimental resources, construction of experimental teaching teams and other aspects. In addition, the goal of Shanghai Business School is to construct a virtual simulation experimental center with the industry characteristics of commercial circulation, multi-layered experimental system, intelligent open management, modern virtual simulation experimental technology and teaching staff integrating industry, education and research.

\subsection{Design of virtual simulation experimental teaching system}

With the deepening of transformation and reform of applied-oriented undergraduate universities, the center relies on the development trend of modern commercial circulation industry, and centers on the layout planning of the school prioritizing the development of disciplines and majors related to business operation and management and business culture construction. It integrates the experimental teaching resources of three key disciplines including business administration, business economics and business culture communication and support disciplines including business language, e-commerce, computer technology and so on, and forms a "three-level, four-module and four-mode" virtual simulation experimental teaching system in the construction of experimental teaching system and experimental content.

\subsection{Co-construction by university-enterprise cooperation}

Through cooperating with enterprises to construct virtual simulation experimental teaching, the center can implant the successful business examples of enterprises into experimental teaching activities and make the content of experimental teaching closer to reality. In addition, the center can take advantage of practical research and development capability of enterprises to develop a lot of real business data, and mature comprehensive practical projects and improve experimental teaching effect. Furthermore, the center can effectively combine on-campus analog simulation experimental resources with off-campus practical resources and achieve the co-construction effect of combining virtuality with reality and complementing each other's advantages through the participation of enterprises in constructing on-campus laboratories and cooperation with new practice base co-constructed by universities and enterprises.

\subsection{Sharing of teaching resources}

At the beginning of construction, Modern Commercial Circulation Virtual Simulation Experimental Center made clear its construction thought, namely "co-construction, sharing and win-win situation". "Co-construction" mainly embodies multiple construction modes of experimental platforms of the center, including co-construction with enterprises, teaching and scientific research bases and so on. "Sharing" means that the center is open to the outside world after completion, serves the society, radiates peripheral small and medium-sized enterprises and universities and realizes the regional sharing of resources, information, construction experience and 
achievements. "Win-win situation" refers to sharing achievements after the construction of the center and realizing the common benefits of the society, enterprises, schools, teachers, students and staff.

\subsection{Construction of teaching platforms}

The center spares no effort to push forward "comprehensive and integrated construction of smart campus" integrating the software and hardware condition construction of cloud computing center, integrated command and control system, comprehensive and integrated service intelligent management software and hardware system, mobile Internet and mobile service application app, practical teaching platforms related to the cross-school operation and management of cloud technology and smart university to serve the connotation construction of the school, enhance the convenience degree of teaching and scientific research for teachers and learning life for students and promote the scientific and efficient daily management of the school.

\section{Characteristics and innovation of modern circulation virtual simulation experimental teaching centers}

(1) Embody the collaborative development trend of Internet technology and commercial forms and construct virtual simulation experimental modules with modern business technology.

(2) Depend on virtual simulation experimental platforms and establish the cross-school sharing mechanism of superior experimental resources.

(3) Gain experimental content from business application practice and transformation of scientific research achievements and aim to improve application ability and cultivate innovation ability.

(4) Insist on the experimental teaching principle of "combining virtuality with reality, complementing each other and emphasizing reality rather than virtuality" and promote the construction of teaching platforms in virtual business practice environment.

\section{References}

[1] Wang Weiguo, Thoughts and Suggestions on the Construction of Virtual Simulation Experimental Teaching Centers, Research and Exploration in Laboratory, 2013, 32 (12): 5-8.

[2] Zhang Hongxia, Construction of Virtual Simulation Experimental Teaching Centers of Disciplines Related to Economic Management in Universities, Journal of Higher Education, 2015 (18): 238-239.

[3] Huang Qi, Thoughts on the Construction of Analog Simulation Experimental Teaching System in Universities Related to Economic Management, Journal of Seeking Knowledge Guide, 2015 (7): 61-61.

[4] Liu Qin, Thoughts on the Construction of Experimental Teaching System Related to Economic Management in Universities, Economic \& Trade Update, 2012 (18): 243-243. 